\title{
Produção e caracterização de compósitos de concreto com adição de pneus automotivos
}

\author{
Production and characterizaration of concrete composites with addition of automotive tyres
}

Rafael Camargo Cardoso ${ }^{+*}$ e Ricardo de Freitas Cabral *

Como citar esse artigo. Cardoso, GN e Cabral, RF. Produção e caracterização de compósitos de concreto com adição de pneus automotivos. Revista Teccen. 2017 Jun/Dez.; 10 (2): 18-23

\begin{abstract}
Resumo
O presente trabalho teve como motivador o crescimento exponencial do número de pneus descartados inadequadamente no meio ambiente assim como os impactos ambientais que os mesmos causam para o meio. Tem-se como objetivo principal introduzir pneus com granulometrias similares a da areia na confecção do cimento para a construção de pisos. Para tal utilizou-se como referencia um matriz onde não havia percentual de agregado para à partir desses resultados fazermos os comparativos com compósitos de medidas pré-estabelecidas de adição do pneu (5 e 10\%). Como instrumentos para análise comparativa foi feito o uso dos testes de compressão axial e de Microscopia Eletrônica de Varredura (MEV). Com os resultados obtidos com o teste de compressão é possível descrever que quando mais borracha adicionada á matriz maior a perda de Tensão da mesma, ou seja, a adição de borracha é inversamente proporcional a Tensão. Com base nas análises obtidas com o Microscópio Eletrônico de Varredura, conclui-se que não há uma perfeita homogeneização entre a matriz e o reforço devido à geometria do reforço, sendo assim há a presença de lacunas que apresentam baixo desempenho para o material.

Palavras-Chave: Compósito; Pneu automotivo; Reciclagem
\end{abstract}

\begin{abstract}
The present study had as motivator the exponential growth of the number of tires discarded inadequately in the environment as well as the environmental impacts that they cause for the environment. Its main objective is to introduce tires with granulometry similar to sand in the construction of cement for the construction of floors. For this purpose, a matrix was used where there was no percentage of aggregate to make the comparisons with composites of pre-established tire addition measures (5 and 10\%). As instruments for comparative analysis, the use of axial ompression tests and Scanning Electron Microscopy (SEM). With the results obtained with the compression test it is possible to describe that when more rubber added to the matrix the greater the loss of Voltage of the same, in other words, the addition of rubber is inversely proportional to Voltage. Based on the analyzes obtained with the Scanning Electron Microscope, it is concluded that there is no perfect homogenization between the matrix and the reinforcement due to the geometry of the reinforcement, so there are gaps that have low performance for the material.

Keywords: Automotive tyre; Composite; Recycling.
\end{abstract}

\section{Introdução}

Atualmente, a busca por meios e/ou medidas que minimizem os impactos ambientais na sociedade vem sendo um dos principais objetivos de todas as organizações seja ela de qualquer esfera, porte e ramo ao qual desenvolve seus produtos.

Preocupações com os impactos ambientais vão muito além de normas e legislações que rege as organizações, estar atento a questões ligadas ao meio ambiente faz com que toda e qualquer empresa tenha uma visão totalmente diferenciada quanto as demais inseridas no mercado, e não é só isso, podem a partir de suas próprias atitudes trazer inúmeros outros benefícios, tais como: redução e impostos, minimização dos custos, reutilização de materiais que antes eram descartados, entre outros. De acordo com Filho et al. (2015), há três razões principais que as empresas visam quando o assunto é a aplicabilidade da Logística Reversa: (1) Legislação Ambiental, a preocupação em cuidar e dar o tratamento necessário dos seus produtos depois do uso atendendo as leis que normatizam tais seguimentos; (2) Ganhos financeiros com a reinserção de seus produtos na linha de produção e; (3) a maximização e a percepção dos meios internos da visibilidade e importância com q a empresa dá para questões ligadas a conscientização ambiental.

Afiliação dos autores: † Discente da UNIFOA - Centro Universitário de Volta Redonda, Volta Redonda, Rio de Janeiro.

† Docente da UNIFOA - Centro Universitário de Volta Redonda, Volta Redonda, Rio de Janeiro.

* E-mail para correspondência: rafaelkamargo@hotmail.com 
Uma das principais maneiras, dentre as diversas, de se adequar a esta necessidade, o reaproveitamento de materiais é a que se adequa melhor aos tempos atuais, ou seja, a logística reversa se faz o mais eficiente, pois além de agregar propriedades de todas as esferas possíveis, faz com que as organizações passem a atender as normas relacionadas ao destino ecologicamente correto de resíduos.

Diversos são os trabalhos na área de adição do pneu automotivo em diferentes aplicabilidades, como por exemplo, em asfaltos, tubos de concretos em cerâmicos voltados para a construção civil, apresentados por outros profissionais que contribuíram significativamente para o desenvolvimento deste, tais como, Dos Santos (2012), Queiroz (2012) e Santos (2012).

O presente trabalho tem por finalidade a verificação de propriedades mecânicas e morfológicas de vigas de concreto com adições de resíduos de pneus reciclados. Tais agregados não substituirão quaisquer materiais necessários para a confecção das vigas tradicionais, mas serão adicionados na composição final do compósito, em medidas de $5 \%$ e $10 \%$.

\section{Material e métodos}

Para a preparação do compósito foram utilizados os seguintes materiais: cimento Portland CP III (NBR 5735/91), areia média (NBR 7225/ 93), brita 1( NBR 7225/93), água e agregado moído de pneu. Com objetivo de avaliar se os materiais utilizados estavam realmente de acordo com suas normas regulamentadoras, a brita e a areia, passaram pela análise de granulometria onde a partir da passagem pelas peneiras no agitador de peneiras (Lab1000/Solotest) por 10 minutos, foi possível comprovar que os materiais utilizados estavam de acordo com as normas regulamentadoras. Para a utilização do pneu submeteu-se o material no mesmo processo da brita e areia, porém para a separação fez-se o uso das mesmas granulometrias da areia média $(1,2+0,42 \mathrm{~mm} / \mathrm{NBR} 7225 / 93)$.

Para a confecção dos corpos de provas foras utilizados como referencias, os trabalhos dos seguintes autores, Silva Junior et al. (2014), Romualdo et al (2011), devido a similaridade entre as pesquisas já realizadas com a atual pesquisa. E como referencias técnicas as normas regulamentadoras NBR 5738/03 e NBR 5739/07

Os moldes utilizados para a confecção dos corpos de provas seguem as especificações estabelecidas pela NBR 5738/03, onde esta descreve que o molde tem que ter sua área em tordo de 78,57 $\mathrm{cm}^{2}$, podendo o diâmetro exceder em $1 \%$ e a altura em $2 \%$, nos casos dos moldes cilíndricos.

Para a realização dessa pesquisa foram desenvolvidos 12 corpos de prova, para o teste de Compressão axial, com idades de 28 e também foram realizadas análises por $\mathrm{MEV}$, com os corpos de prova ensaiados.

Quanto à preparação do concreto foi utilizado às medias previstas na NBR 12655/06, que possuem as seguintes características de traços: 1 (cimento), 2 (área) e 3 (brita), onde a quantidade estimada dos materiais do traço gira em torno de 5 litros de cimento, 10 litros de areia, 15 litros de brita e 3,3 litros de água. A borracha foi adicionada nas medidas de $5 \%$ e $10 \%$ da medida do concreto areia. A Tabela 1 apresenta o quantitativo em litros por traço.

Para a preparação do concreto de $10 \%$ fez-se necessário o acréscimo de $27 \%$ de água para que fosse possível obter a textura ideal do compósito. Este acréscimo acredita-se que ocorreu devido à dificuldade da homogeneização da borracha com os demais materiais.

A análise morfológica desta pesquisa foi realizada no MEV da marca Hitachi modelo TM3000, por meio de um detector de elétrons secundários, onde foram analisadas a morfologia interna das vigas oriundas dos ensaios de compressão axial.

\section{Resultados e Discussão}

O ensaio de compressão axial dividiu-se conforme o tempo de análise pré-estabelecida, sendo analisada cada uma das proporções utilizadas para a confecção dos corpos de provas, apenas para analise padrão, para esse estudo utilizou-se apenas os resultados dos corpos de provas com 28 dias de acordo com as referências utilizadas (NBR 5739/07). A Figura 1 descreve os resultados obtidos a partir do ensaio com os corpos de provas no vigésimo oitavo dia de cura.

A Figura 2, apresentaum comparativo entre a Tensão e o teor de borracha dos corpos de provas. É possível descrever que os corpos de provas apresentam indicadores inferiores a matriz referencia que apresenta $0 \%$ de adição de pneu, porém os corpos de provas com $5 \%$ são o que apresentam valores mais próximos. A análise conclui que a comparação da média dos moldes com $5 \%$ apresenta uma perda de aproximadamente $8 \%$ quando comparado com a matriz já a média dos de $10 \%$ de adição de borracha descrevem uma perda de 
quase $20 \%$ de sua tensão. Autores como Junior et al. (2016), Romualdoet al. (2011), Santos et al. (2004) e Nirschl (2002), apresentam em suas pesquisas resultados bem similares aos encontrados. Santos et

Tabela 1 - Percentual de matéria prima.

\begin{tabular}{|c|c|c|c|c|}
\hline Percentual & Cimento & Brita & Areia & Borracha \\
\hline $\mathbf{0 \%}$ & 5 & 15 & 10 & 0 \\
\hline $\mathbf{5 \%}$ & 5 & 15 & 10 & 1,0 \\
\hline $\mathbf{1 0 \%}$ & 5 & 15 & 10 & 2,0 \\
\hline
\end{tabular}

\begin{tabular}{|c|c|c|c|c|c|c|c|c|c|c|c|c|c|}
\hline & \multicolumn{3}{|c|}{ CP1 } & \multicolumn{3}{|c|}{ CP2 } & \multicolumn{3}{|c|}{ CP3 } & Média & Média & Desvio & Desvio \\
\hline & $\stackrel{0}{0}$ & 홍글 & 氮 & 焉 & 한? & 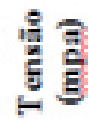 & 要 & 한? & 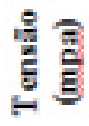 & 형ㅇ & 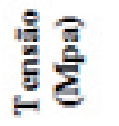 & 홓의 & 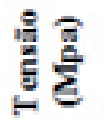 \\
\hline $0 \%$ & 78,54 & 201.8 & 25,6 & 78,54 & 200.4 & 25,5 & 78,54 & 205.3 & 26,1 & 202.5 & 25.7 & - & - \\
\hline $5 \%$ & 78,54 & 181.5 & 23,1 & 78,54 & 177.1 & 22,5 & 78,54 & 202.5 & 25,7 & 187.03 & 23,7 & 15.47 & 2,0 \\
\hline $10 \%$ & 78,54 & 163.8 & 20,8 & 78,54 & 165.9 & 21,1 & 78,54 & 161.7 & 20,5 & 163.8 & 20,8 & 38.7 & 4,9 \\
\hline
\end{tabular}

Figura 1- Resultados do ensaio de compressão. Autoria própria.

al (2004) mesmo fazendo uso de aditivos descrevem as perdas similares a dessa pesquisa porém conseguem minimiza-las, segundo os autores"podese verificar que a ação do aditivo é preponderante para minimizar as perdas para pequenos teores de borracha".

As imagens da amostra deste trabalho são apresentadas nas Figuras 4 (a) e (b),5 (a) e (b),6 (a) e (b).Com os resultados obtidos da análise do MEV, descreve-se que a relação entre a matriz e o reforço não atinge com eficiência a sua homogeneização, o que acarreta na minimização das propriedades do material. Observa-se também que nos corpos de provas com adição de pneu há a presença de espaços o que reduz algumas propriedades desses materiais, este resultado também foi obtido por Souza et al (2014). O autor ainda descreve que esses espaços e trincas reduzem significativamente a resistência do material.

\section{Conclusão}

Nos concretos onde foram adicionados os resíduos de borracha quando comparado com a matriz, à resistência a compressão teve uma queda

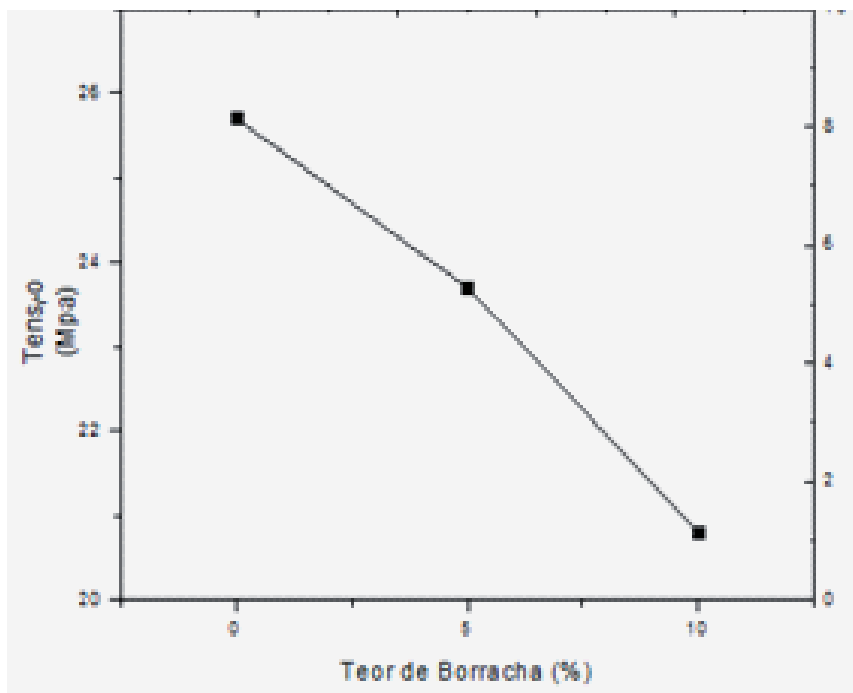

Figura 2. Comportamentos da resistência a compressão. Autoria própria.

bem significativa, onde os corpos de prova de $5 \%$ apresentaram $8 \%$ de perda e os de $10 \%$ reduziram aproximadamente $20 \%$.

Os resultados do ensaio de compressão mostra que não há uma razão fixa entre o quantitativo de adição de borracha e a perda de sua resistência, uma vez que a proporção de borracha utilizada é o dobro e o mesmo não acontece com a perda da 


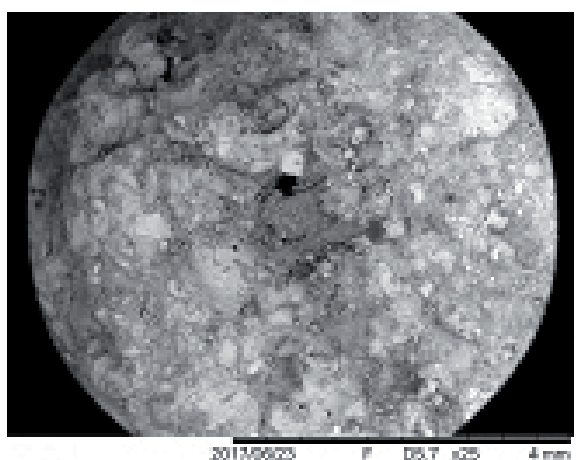

Figura 3(a)- Imagem estrutural 25x (Matriz).

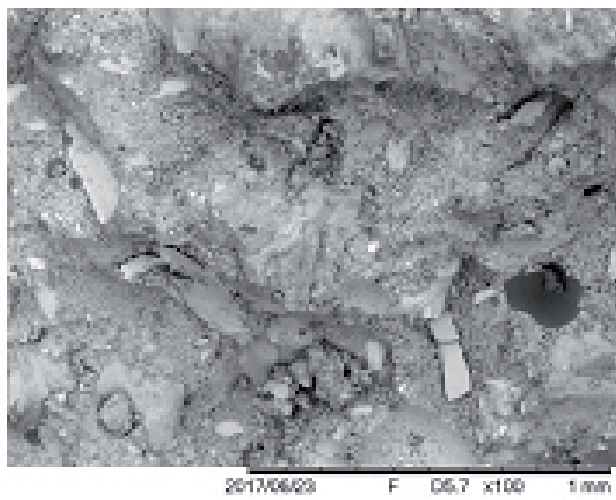

Figura 4(a)- Imagem estrutural 100x (5\%).

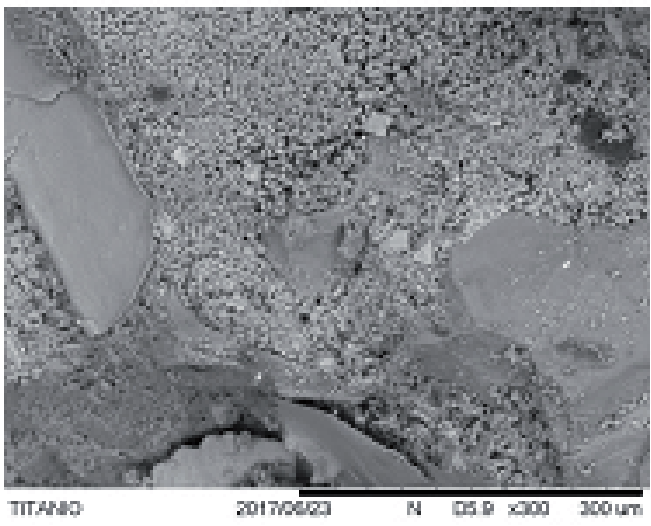

Figura 5(a)- Imagem estrutural 300x (10\%).

propriedade, por sinal é ainda mais elevado, cerca de $12 \%$ quando comparado os resultados com $5 \%$ adição.

As imagens de MEV mostram que não há uma homogeneização total entre os elementos da matriz com o pneu, pressupõem-se a partir das análises que mesmo não havendo essa prefeita homogeneização o pneu agrega propriedades no produto final.Concluise com base nos teste realizados que há a viabilidade

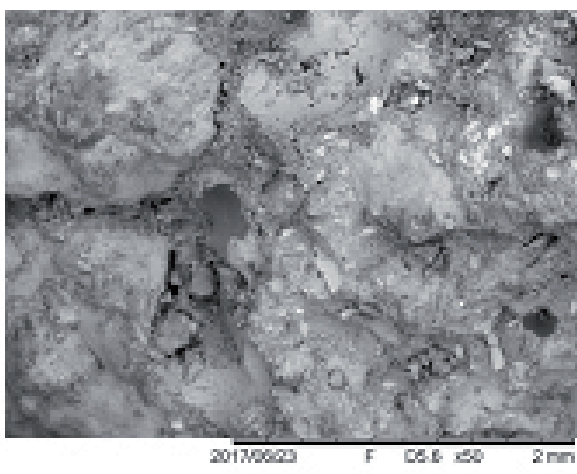

Figura 3(b)- Imagem estrutural 50x (Matriz).

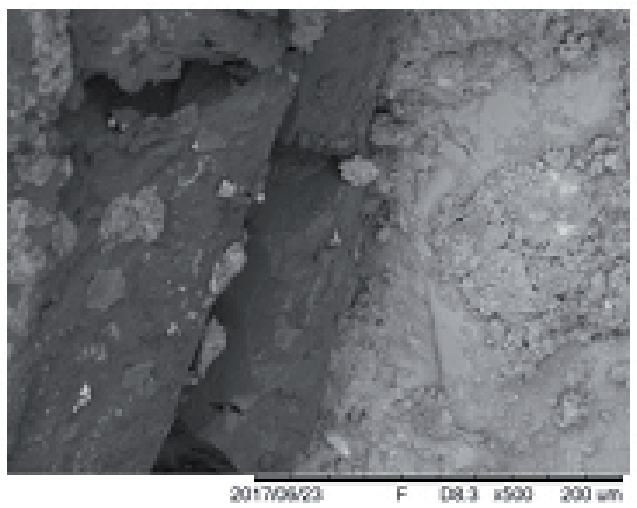

Figura 4(b)- Imagem estrutural 500x (5\%).

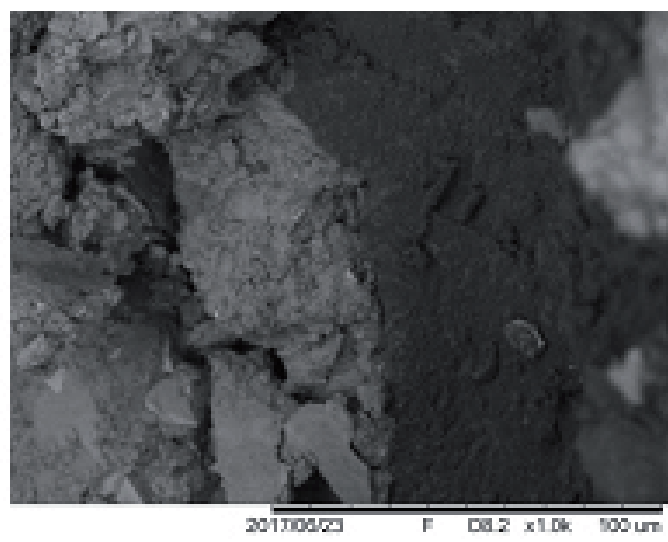

Figura 5(b)- Imagem estrutural 1000x (10\%).

da aplicação da borracha (5\%) na confecção do concreto, apesar de apresentarem perda de tensão, estes apresentam características para possíveis produtos que não apresentam principal objetivo o recebimento de cargas em pontos específicos apenas cargas distribuídas, não terão fragilidade como a apresentada nos testes, tais produtos como, por exemplo, pisos inter travados.

Outros aspectos que contribuem 
significativamente para a aplicação desta pesquisa são o impacto ambiental que o agregado representa no meio ambiente, a redução do consumo das fontes naturais e também a menor taxa de emissão atmosférica de alguns poluentes provenientes da queima do pneu.

Propõe-se para trabalhos futuros o estudo da viabilidade da aplicação do concreto para a produção de pisos inter travados assim como o teste de abrasão, teste esse que apresentaria grande resultados para esse estudo, pois tem o objetivo de mensurar os desgastes que os mesmos apresentariam.

\section{Referências}

ABNT - ASSOCIAÇÃO BRASILEIRA DE NORMAS TÉCNICA . (1991). NBR 5735: Cimento Portland comum. Rio de Janeiro, RJ.

ABNT - ASSOCIAÇÃO BRASILEIRA DE NORMAS TÉCNICAS. (1993). NBR 7225: Materiais de pedra agregados naturais. Rio de Janeiro, RJ.

ABNT - ASSOCIAÇÃO BRASILEIRA DE NORMAS TÉCNICAS. (2003). NBR5738: Moldagem e Cura de corpos-de-prova cilíndricos ou prismáticos de concreto. Rio de Janeiro, RJ.

ABNT - ASSOCIAÇ̃̃O BRASILEIRA DE NORMAS TÉCNICAS. (2003). NBR5739: Concreto - Ensaio de compressão de corpos-de-prova cilíndricos. Rio de Janeiro, RJ.

DOS SANTOS, L. E. M. (2016). ESTUDO DE ESTRUTURA PRÉMOLDADA COM ADIĈ̃̃O DE RESÍDUO DE PNEU - (Dissertação de Mestrado não publicada). Universidade Federal do Pará. Belém, PA.

Thode Filho, S., Machado, C. J. S., Vilani, R. M., Paiva, J. L., \& Costa Marques, M. R. (2015). A logística reversa e a Política Nacional de Resíduos Sólidos: desafios para a realidade brasileira. Electronic Journal of Management, Education and Environmental Technology (REGET), 19(3), 529-538.

Nirschl, G. C.,Akasaki, J.L., \& Fioriti, C. F. (2002). Influência da granulometria das fibras de borracha vulcanizada em dosagens de concreto. In Annais: $44^{\circ}$ Congresso Brasileiro do Concreto. Belo Horizonte: IBRACON..

Queiroz, R. M. (2012). Tubos de concreto com adição de resíduos de borracha de pneu. (Dissertação mestrado), Faculdade de de Ilha Solteira - Engenharia, Universidade Estadual Paulista. Ilha Solteira - São Paulo.

Romualdo, A. C. A., Santos, D. E., CASTRO, L., Menezes, W. P., Pasqualetto, A., \& Santos, O. R. (2011). Pneus inservíveis como agregados na composição de concreto para calçadas de borracha. In: 3rd International workshop advances in cleaner production. São Paulo.

Santos, A. C. D. (2005). Avaliação do comportamento do concreto de borracha obtida a partir da reciclagem em placas pré-moldadas. (Dissertação de Mestrado em Engenharia Civil), Universidade

Federal de Alagoas. Maceió, Alagoas.

Santos, R., Barboza, A. D. S. R., GOMES, P., \& Lima, F. (2004). Avaliação do comportamento do concreto com adição de borracha obtida a partir da reciclagem de pneus para aplicação em elementos pré-moldados. I Conferência Latino-Americana de Construção Sustentável, X Encontro Nacional de Tecnologia do Ambiente Construído - São Paulo, SP.

Santos, R. M. (2012). Efeito da adição de partículas de borracha de pneus nas propriedades físico-mecânicas de compósito cimentício. (Dissertação de Mestrado). Departamento de Engenharia, Universidade Federal de São João Del-Rei. São João Del-Rei, MG.

Silva Júnior, F. D., \& Martinelli, A. E. (2014). Análise das propriedades do agregado miúdo: resíduo de borracha de pneu para compósitos cimentícios. $21^{\circ} \mathrm{CBECIMAT}$ - Congresso Brasileiro de Engenharia e Ciência dos Materiais, Cuiabá, MT, Brasil.
N.R.G., \& Oliveira, M. L. (2014). Obtenção de um compósito utilizando raspa de pneu para a fabricação de blocos para pavimentação. $21^{\circ}$ CBECIMAT - Congresso Brasileiro de Engenharia e Ciência dos Materiais, Cuiabá, MT, Brasil. 